\title{
FORWARD RATE VOLATILITIES, SWAP RATE VOLATILITIES, AND THE IMPLEMENTATION OF THE LIBOR MARKET MODEL
}

\author{
John Hull and Alan White* \\ Joseph L. Rotman School of Management \\ University of Toronto \\ 105 St George Street \\ Toronto, Ontario M5S 3E6 \\ Canada
}

Tel: (416) 9788615 (Hull)

Tel: (416) 9783689 (White)

Fax: (416) 5157559

e-mails: hull@mgmt.utoronto.ca; awhite@mgmt.utoronto.ca

*We are grateful to Lief Andersen, Jesper Andreasen, Robert Engle, Steve Figlewski, Josh
Rosenberg, and Marti Subrahmanyam for comments on an earlier version of this paper. 


\title{
FORWARD RATE VOLATILITIES, SWAP RATE VOLATILITIES, AND THE IMPLEMENTATION OF THE LIBOR MARKET MODEL
}

\begin{abstract}
This paper presents a number of new ideas concerned with the implementation of the LIBOR market model and its extensions. It develops and tests an analytic approximation for calculating the volatilities used by the market to price European swap options from the volatilities used to price interest rate caps. The approximation is very accurate for the range of market parameters normally encountered and enables swap option volatility skews to be implied from cap volatility skews. It also allows the LIBOR market model to be calibrated to broker quotes on caps and European swap options so that other interest rate derivatives can be valued.
\end{abstract}


In recent years a new model for valuing interest rate derivatives has been developed by Brace, Gatarek, and Musiela (1997), Jamshidian (1997), and Miltersen, Sandmann, and Sondermann (1997). It is usually referred to as the LIBOR market model. It is an extension of the well-known Heath, Jarrow, and Morton (HJM) (1992) model. Whereas the HJM model describes the behavior of instantaneous forward rates expressed with continuous compounding, the LIBOR market model describes the behavior of the forward LIBOR rates underlying caps and floors, with the usual market conventions being used for compounding (that is, the compounding period equals the tenor of the rate). The main advantage of the LIBOR market model over HJM is that it easier to calibrate the model to the market prices of interest rate caps and European swap options. Also, as shown by Brace, Gatarek, and Musiela (1997), the LIBOR market model overcomes some technical existence problems associated with the lognormal version of the HJM model.

In this paper we explain the LIBOR market model and describe how it can be implemented. We use the model to develop and test a new analytic approximation for pricing European swap options. We show how this approximation makes it possible to translate the volatility skews observed for caps into volatility skews for European swap options. We also explain how it can be used to simplify the process of calibrating the LIBOR market model to the market prices of caps and swap options. 


\section{THE STANDARD MARKET MODELS FOR CAPS AND SWAPTIONS}

The most popular over-the-counter interest rate options are interest rate caps/floors and European swap options. The standard market models for valuing these instruments are versions of Black's (1976) model. This model was originally developed for valuing options on commodity futures, but has found many other applications in financial engineering. When Black's model is used to value a caplet (one element of an interest rate cap), the underlying interest rate is assumed to be lognormal. When it is used to value European swap options, the underlying swap rate is assumed to be lognormal. Researchers such as Jamshidian (1997) have shown that the cap/floor market model and the European swap option market model are each internally consistent in the sense that they do not permit arbitrage opportunities. However, they are not consistent with each other.

In this section we explain the standard market models for pricing caps and European swap options. This will form a useful background for our discussions of the LIBOR market model in later sections. We will use the result of Harrison and Kreps (1979) that, in any market where there is no arbitrage, for any given numeraire security whose price is $g(t)$, there exists a measure for which $f(t) / g(t)$ is a martingale for all security prices $f(t)$. The measure will be denoted by $\mathcal{M}\{g(t)\}$.

\section{Interest Rate Caps}

Consider a cap with strike rate $R_{c}$ and reset dates at times $t_{1}, t_{2}, \ldots, t_{N}$ with a final payment date $t_{N+1}$. Define $\delta_{i}=t_{i+1}-t_{i}$ and $R_{i}$ as the $\delta_{i}$-maturity rate observed at time $t_{i}$ and expressed with a compounding period of $\delta_{i}(1 \leq i \leq N)$. The cap is a portfolio of $N$ caplets. The $i$ th caplet provides a payoff at time $t_{i+1}$ equal to

$$
L_{c} \delta_{i} \max \left[R_{i}-R_{c}, 0\right]
$$

where $L_{c}$ is the principal.

Define $P(t, T)$ as the price of a discount bond paying off $\$ 1$ at time $T$. To value the 
$i$ th caplet we use $P\left(t, t_{i+1}\right)$ as the numeraire. Under the measure, $\mathcal{M}\left\{P\left(t, t_{i+1}\right)\right\}$

$$
\frac{f(t)}{P\left(t, t_{i+1}\right)}
$$

is a martingale for all security prices $f(t)$. Hence

$$
\frac{f(0)}{P\left(0, t_{i+1}\right)}=E_{i+1}\left[\frac{f(t)}{P\left(t, t_{i+1}\right)}\right]
$$

where $E_{i+1}$ denotes expectations under $\mathcal{M}\left\{P\left(t, t_{i+1}\right)\right\}$.

By setting $f(t)=P\left(t, t_{i}\right)-P\left(t, t_{i+1}\right)$ in equation (2), we see that

$$
F_{i}(0)=E_{i+1}\left(R_{i}\right)
$$

where $F_{i}(t)$ is the forward interest rate for the period $\left(t_{i}, t_{i+1}\right)$, expressed with a compounding period $\delta_{i}$, observed at time $t$. Equation (3) shows that, under the assumed measure, the value at time zero of this forward rate equals the expected future spot rate for $\left(t_{i}, t_{i+1}\right)$.

By setting $f(t)$ equal to the price of the $i$ th caplet and noting that $P\left(t_{i+1}, t_{i+1}\right)=1$, we see from equation (2) that

$$
f(0)=P\left(0, t_{i+1}\right) E_{i+1}\left[f\left(t_{i+1}\right)\right]
$$

or

$$
f(0)=P\left(0, t_{i+1}\right) L_{c} \delta_{i} E_{i+1}\left[\max \left(R_{i}-R_{c}, 0\right)\right]
$$

Assuming $R_{i}$ is lognormal, with the standard deviation of $\ln \left(R_{i}\right)$ equal to $\sigma_{i} \sqrt{t_{i}}$, this becomes

$$
f(0)=P\left(0, t_{i+1}\right) L_{c} \delta_{i}\left[E_{i+1}\left(R_{i}\right) N\left(d_{1}\right)-R_{c} N\left(d_{2}\right)\right]
$$

where

$$
\begin{gathered}
d_{1}=\frac{\ln \left[E_{i+1}\left(R_{i}\right) / R_{c}\right]+\sigma_{i}^{2} t_{i} / 2}{\sigma_{i} \sqrt{t_{i}}} \\
d_{2}=d_{1}-\sigma_{i} \sqrt{t_{i}}
\end{gathered}
$$


and $N(x)$ is the cumulative normal distribution function. Substituting from equation (3) gives the standard market model for valuing the caplet:

$$
f(0)=P\left(0, t_{i+1}\right) L_{c} \delta_{i}\left[F_{i}(0) N\left(d_{1}\right)-R_{c} N\left(d_{2}\right)\right]
$$

where

$$
\begin{gathered}
d_{1}=\frac{\ln \left[F_{i}(0) / R_{c}\right]+\sigma_{i}^{2} t_{i} / 2}{\sigma_{i} \sqrt{t_{i}}} \\
d_{2}=d_{1}-\sigma_{i} \sqrt{t_{i}}
\end{gathered}
$$

Similarly the standard market model for valuing the $i$ th element of a floor is

$$
f(0)=P\left(0, t_{i+1}\right) L_{c} \delta_{i}\left[R_{c} N\left(-d_{2}\right)-F_{i}(0) N\left(-d_{1}\right)\right]
$$

The variable $\sigma_{i}$ is often referred to the volatility of the $\left(t_{i}, t_{i+1}\right)$ forward rate. In fact, there is no requirement that the volatility of this forward rate be constant during the life of the caplet. All that is required is that the variable $\sigma_{i}^{2}$ be the average variance rate of the forward rate during the life of the caplet. This point is important for understanding of the model in Section II. To avoid confusion with variables introduced in Section II, we will refer to $\sigma_{i}$ as the spot volatility of the $\left(t_{i}, t_{i+1}\right)$ rate, or the spot volatility of the $i$ th caplet.

In practice brokers usually quote what is known as a flat volatility for a cap. This is a volatility which, if used as the spot volatility for all the underlying caplets, reproduces the cap's market price. When flat volatilities for all cap maturities are available or can be estimated, spot volatilities can be calculated. The procedure is to use the flat volatilities and equation (4) to calculate cap prices for a particular strike rate, deduce caplet prices by subtracting one cap price from the next, and then imply the spot volatilities from these caplet prices using equation (4).

\section{European Swap Options}

We now move on to consider a European swap option. Suppose that the underlying swap lasts from time $t_{n}$ to time $t_{N+1}$ and the strike rate is $R_{s}$. The swap reset dates are $t_{n}$, 
$t_{n+1}, \ldots, t_{N}$ and the corresponding payment dates are $t_{n+1}, t_{n+2}, \ldots, t_{N+1}$, respectively. We define

$$
A_{n, N}(t)=\sum_{i=n}^{N} \delta_{i} P\left(t, t_{i+1}\right)
$$

where as before $P(t, T)$ is the price of a discount bond paying off $\$ 1$ at time $T$ and $\delta_{i}=t_{i+1}-t_{i}$. We will denote by $S_{n, N}(t)\left(0 \leq t \leq t_{n}\right)$ the forward swap rate. This is the fixed rate which, when it is exchanged for floating in a forward start swap, causes the value of the swap to be zero. An expression for $S_{n, N}(t)$ is

$$
S_{n, N}(t)=\frac{P\left(t, t_{n}\right)-P\left(t, t_{N+1}\right)}{A_{n, N}(t)}
$$

Define $R_{n, N}=S_{n, N}\left(t_{n}\right)$ as the swap rate observed at time $t_{n}$. Both $R_{n, N}$ and $R_{s}$ are expressed with a compounding frequency reflecting the number of swap payments per year. A European swap option where the holder has the right to pay fixed and receive floating can be viewed as providing payments of

$$
L_{s} \delta_{i} \max \left(R_{n, N}-R_{s}, 0\right)
$$

at times $t_{i+1}$ for $n \leq i \leq N$ where $L_{s}$ is the swap principal. The value of the swap option at time $t_{n}$ is therefore

$$
L_{s} A_{n, N}\left(t_{n}\right) \max \left(R_{n, N}-R_{s}, 0\right)
$$

We will value the European swap option at time zero using $A_{n, N}(t)$ as the numeraire. Under the measure $\mathcal{M}\left\{A_{n, N}(t)\right\}$

$$
\frac{f(t)}{A_{n, N}(t)}
$$

is a martingale for all security prices $f(t)$. Hence

$$
\frac{f(0)}{A_{n, N}(0)}=E_{A}\left[\frac{f\left(t_{n}\right)}{A_{n, N}\left(t_{n}\right)}\right]
$$

where $E_{A}$ denotes expectations under the measure. By setting $f(t)=P\left(t, t_{n}\right)-P\left(t, t_{N+1}\right)$ in equation (8) we see that

$$
S_{n, N}(0)=E_{A}\left[S_{n, N}\left(t_{n}\right)\right]=E_{A}\left(R_{n, N}\right)
$$


The forward swap rate, therefore, equals the expected future swap rate under the measure.

We now set $f(t)$ equal to the value of the swap option. Equation (8) gives

$$
f(0)=A_{n, N}(0) E_{A}\left[\frac{f\left(t_{n}\right)}{A_{n, N}\left(t_{n}\right)}\right]
$$

Substituting for $f\left(t_{n}\right)$ from equation (7) we obtain

$$
f(0)=L_{s} A_{n, N}(0) E_{A}\left[\max \left(R_{n, N}-R_{s}, 0\right)\right]
$$

Assuming $R_{n, N}$ is lognormal with the standard deviation of $\ln \left(R_{n, N}\right)$ equal to $\sigma_{n, N} \sqrt{t_{n}}$ this becomes

$$
f(0)=L_{s} A_{n, N}(0)\left[E_{A}\left(R_{n, N}\right) N\left(d_{1}\right)-R_{s} N\left(d_{2}\right)\right]
$$

where

$$
\begin{gathered}
d_{1}=\frac{\ln \left[E_{A}\left(R_{n, N}\right) / R_{s}\right]+\sigma_{n, N}^{2} t_{n} / 2}{\sigma_{n, N} \sqrt{t_{n}}} \\
d_{2}=d_{1}-\sigma_{n, N} \sqrt{t_{n}}
\end{gathered}
$$

Substituting from equation (9) gives the standard market model for valuing the swap option

$$
f(0)=L_{s} A_{n, N}(0)\left[S_{n, N}(0) N\left(d_{1}\right)-R_{s} N\left(d_{2}\right)\right]
$$

where

$$
\begin{gathered}
d_{1}=\frac{\ln \left[S_{n, N}(0) / R_{s}\right]+\sigma_{n, N}^{2} t_{n} / 2}{\sigma_{n, N} \sqrt{t_{n}}} \\
d_{2}=d_{1}-\sigma_{n, N} \sqrt{t_{n}}
\end{gathered}
$$

Similarly the standard market model for valuing a swap option that gives the holder the right to receive fixed and pay floating is

$$
f(0)=L_{s} A_{n, N}(0)\left[R_{s} N\left(-d_{2}\right)-S_{n, N}(0) N\left(-d_{1}\right)\right]
$$

We will refer to $\sigma_{n, N}$ as the spot volatility of the swap rate or the spot volatility of the European swap option. As in the case of interest rate caps, we emphasize that the model does not require the forward swap rate's volatility to be constant between times 0 and $t_{n}$. It is sufficient for its average variance rate to be $\sigma_{n, N}^{2}$ during this period. 


\section{THE LIBOR MARKET MODEL}

In this section we discuss the development and implementation of the LIBOR market

model. Our notation is consistent with Section I. We consider a cap with reset dates at times $t_{1}, t_{2}, \ldots, t_{n}$ and a final payment date $t_{n+1}$. We define $t_{0}=0, \delta_{i}=t_{i+1}-t_{i}$ $(0 \leq i \leq n)$, and

$F_{i}(t)$ : Forward rate observed at time $t$ for the period $\left(t_{i}, t_{i+1}\right)$, expressed with a compounding period of $\delta_{i}$

$P(t, T)$ : Price at time $t$ of a zero-coupon bond that provides a payoff of $\$ 1$ at time $T$

$m(t)$ : Index for the next reset date at time $t$. This means that $m(t)$ is the smallest integer such that $t \leq t_{m(t)}$.

$p$ : Number of factors

$\zeta_{i, q}: q$ th component of the volatility of $F_{i}(t)(1 \leq q \leq p)$

$v_{i, q}: q$ th component of the volatility of $P\left(t, t_{i}\right)(1 \leq q \leq p)$

We assume that the components to the volatility are independent. (This is not restrictive since they can be orthogonalized.) The processes followed by $F_{i}(t)$ and $P\left(t, t_{i}\right)$ are:

$$
\begin{gathered}
d F_{i}(t)=\ldots d t+\sum_{q=1}^{p} \zeta_{i, q}(t) F_{i}(t) d z_{q} \\
d P\left(t, t_{i}\right)=\ldots d t+\sum_{q=1}^{p} v_{i, q}(t) P\left(t, t_{i}\right) d z_{q}
\end{gathered}
$$

where the $d z_{q}$ are independent Wiener processes and the drifts depend on the measure. In this section and the next, we will assume a model where $\zeta_{i, q}(t)$ is a function only of time. The bond price volatility, $v_{i, q}(t)$, is in general stochastic in this model.

We will use as numeraire a money market account that is invested at time $t_{0}$ for a period $\delta_{0}$, reinvested at time $t_{1}$ for a period $\delta_{1}$, reinvested at time $t_{2}$ for a period $\delta_{2}$, and so on. This is equivalent to using a numeraire at time $t$ equal to $P\left(t, t_{m(t)}\right)$. Under the 
chosen measure

$$
\frac{f(t)}{P\left(t, t_{m(t)}\right)}
$$

is a martingale for all security prices $f(t)$ when $t_{m(t)-1} \leq t \leq t_{m(t)}$ so that

$$
\frac{f\left(t_{m(t)-1}\right)}{P\left(t_{m(t)-1}, t_{m(t)}\right)}=E_{m(t)}\left\{\frac{f\left(t_{m(t)}\right)}{P\left(t_{m(t)}, t_{m(t)}\right)}\right\}
$$

or

$$
f\left(t_{m(t)-1}\right)=P\left(t_{m(t)-1}, t_{m(t)}\right) E_{m(t)}\left\{f\left(t_{m(t)}\right)\right\}
$$

where $E_{m(t)}$ denotes expectations under $\mathcal{M}\left\{P\left(t, t_{m(t)}\right)\right\}$ This equation shows that the chosen measure allows us to discount expected values "one accrual period at a time" when security prices are valued. Since cash flows and early exercise opportunities usually occur only on reset dates, this is an attractive feature of the measure.

It can be shown that the $q$ th component of the market price of risk under the measure $\mathcal{M}\left\{P\left(t, t_{j}\right)\right\}$ is $v_{j, q}$ for all $j$. [See for example, Jamshidian (1997).] As shown in Section I, under the measure $\mathcal{M}\left\{P\left(t, t_{i+1}\right)\right\}, F_{i}(t)$ is a martingale so that its drift is zero. It follows that the expected growth rate of $F_{i}(t)$ under the $\mathcal{M}\left\{P\left(t, t_{m(t)}\right)\right\}$ measure is

$$
\sum_{q=1}^{p} \zeta_{i, q}(t)\left[v_{m(t), q}(t)-v_{i+1, q}(t)\right]
$$

so that

$$
\frac{d F_{i}(t)}{F_{i}(t)}=\sum_{q=1}^{p} \zeta_{i, q}(t)\left[v_{m(t), q}(t)-v_{i+1, q}(t)\right] d t+\sum_{q=1}^{p} \zeta_{i, q}(t) d z_{q}
$$

The relationship between forward rates and bond prices is

$$
\frac{P\left(t, t_{j}\right)}{P\left(t, t_{j+1}\right)}=1+\delta_{j} F_{j}(t)
$$

Using this in conjunction with Ito's lemma leads to

$$
v_{j, q}(t)-v_{j+1, q}(t)=\frac{\delta_{j} F_{j}(t) \zeta_{j, q}(t)}{1+\delta_{j} F_{j}(t)}
$$


Repeated application of this result gives

$$
v_{m(t), q}(t)-v_{i+1, q}(t)=\sum_{j=m(t)}^{i} \frac{\delta_{j} F_{j}(t) \zeta_{j, q}(t)}{1+\delta_{j} F_{j}(t)}
$$

and substituting into equation (13) we see that the process followed by $F_{i}(t)$ under the measure $\mathcal{M}\left\{P\left(t, t_{m(t)}\right)\right\}$ is

$$
\frac{d F_{i}(t)}{F_{i}(t)}=\sum_{j=m(t)}^{i} \frac{\delta_{j} F_{j}(t) \sum_{q=1}^{p} \zeta_{j, q}(t) \zeta_{i, q}(t)}{1+\delta_{j} F_{j}(t)} d t+\sum_{q=1}^{p} \zeta_{i, q}(t) d z_{q}
$$

When we take limits allowing the $\delta_{i}$ 's to approach zero, this becomes

$$
d F(t, T)=\sum_{q=1}^{p} \zeta_{q}(t, T) F(t, T) \int_{\tau=t}^{T} \zeta_{q}(t, \tau) F(t, \tau) d \tau+\sum_{q=1}^{p} \zeta_{q}(t, T) F(t, T) d z_{q}
$$

where the notation is temporarily changed so that $F(t, T)$ is the instantaneous forward rate at time $t$ for maturity $T$ and $\zeta_{q}(t, T)$ is the $q$ th component of the volatility of $F(t, T)$. This is the HJM result. The HJM model is, therefore, a limiting case of the LIBOR market model.

\section{Determining Forward Rate Volatilities}

We now simplify the model by assuming that $\zeta_{i, q}(t)$ is a function only of the number of whole accrual periods between the next reset date and time $t_{i}$. Define $\lambda_{j, q}$ as the value of $\zeta_{i, q}(t)$ when there are $j$ such accrual periods. This means that

$$
\zeta_{i, q}(t)=\lambda_{i-m(t), q}
$$

Define $\Lambda_{j}$ as total volatility of a forward rate when there are $j$ whole accrual periods until maturity so that

$$
\Lambda_{j}=\sqrt{\sum_{q=1}^{p} \lambda_{j, q}^{2}}
$$

The $\Lambda$ 's can be in theory be calculated from the spot volatilities, $\sigma_{i}$, used to value caplets in equation (4). Equating variances we must have:

$$
\sigma_{i}^{2} t_{i}=\sum_{j=1}^{i} \Lambda_{i-j}^{2} \delta_{j-1}
$$


so that the $\Lambda$ 's can be obtained inductively. For example, if $\sigma_{1}, \sigma_{2}$, and $\sigma_{3}$ are $20 \%, 22 \%$ and $21 \%$, respectively and the $\delta_{i}$ are all equal, $\Lambda_{0}=20 \%, \Lambda_{1}=23.83 \%$ and $\Lambda_{2}=18.84 \%$

We prefer to calculate the $\Lambda$ 's directly from the flat volatilities quoted by brokers. In the United States brokers quote volatilities for quarterly reset caps with maturities of 1 , $2,3,4,5,7$, and 10 years. A 10-year LIBOR market model with quarterly resets requires that $\Lambda_{j}$ be estimated for $0 \leq j \leq 38$. We assume that $\Lambda_{j}$ is constant for $0 \leq j \leq 2$, for $3 \leq j \leq 6$, for $7 \leq j \leq 10$, for $11 \leq j \leq 14$, for $15 \leq j \leq 18$, for $19 \leq j \leq 26$, and for $27 \leq j \leq 38$. (Given the data available this seems reasonable. The values of $\Lambda_{j}$ for $0 \leq j \leq 2$ determine the price of a one-year cap; these values of $\Lambda_{j}$ together with the values for $3 \leq j \leq 6$ determine the price of the 2-year cap; and so on.) The $\Lambda$ 's are chosen to minimize the sum of the squares of the errors in the flat volatilities implied by the model. To avoid extreme variations in the $\Lambda$ 's and the possibility of negative $\Lambda$ 's, we impose the smoothness condition that the $\Lambda_{j}$ be a non-increasing function of $j$ for $j \geq 11{ }^{1}$

Similarly to Rebonato (1999), we favor a two step approach to obtaining the $\lambda_{j, q}$. The first stage is to determine the $\Lambda_{j}$ from market data as just described; the second stage is to use historical data to determine the $\lambda_{j, q}$ from the $\Lambda_{j}$. Rebonato (1999) proposes a way of determining the $\lambda_{j, q}$ from the $\Lambda_{j}$ to provide as close a fit as possible to the correlation matrix for the $F_{j}(t)(1 \leq j \leq N)$. As Rebonato points out, the approach gives similar results to a principal components analysis. If output from a principal components analysis on the $F_{j}(t)(1 \leq j \leq N)$ is available, we can use it in a direct way to determine values of $\lambda_{j, q}$. The principal components analysis model is

$$
\Delta F_{j}=\sum_{q=1}^{N} \alpha_{j, q} x_{q}
$$

where $\alpha_{i, q}$ is the factor loading for the $i$ th forward rate and the $q$ th factor, $x_{q}$ is the factor score for the $q$ th factor, and

$$
\sum_{j=1}^{N} \alpha_{j, q_{1}} \alpha_{j, q_{2}}
$$


equals 1 when $q_{1}=q_{2}$ and zero when $q_{1} \neq q_{2}$. Define $s_{q}$ is the standard deviation of the $q$ th factor score If the number of factors being used, $p$, were equal to $N$, the number of forward rates, it would be correct to set

$$
\lambda_{j, q}=\alpha_{j, q} s_{q}
$$

for $1 \leq j, q \leq N$. When $p<N$, the $\lambda_{j, q}$ can be scaled so that the relationship in equation (17) holds. This involves setting

$$
\lambda_{j, q}=\frac{\Lambda_{j} s_{q} \alpha_{j, q}}{\sqrt{\sum_{q=1}^{p} s_{q}^{2} \alpha_{j, q}^{2}}}
$$

The analyses reported in this paper are based on a three-factors $(p=3)$. The factors are typical of those obtained from a principal components analysis. The first factor corresponds to a roughly parallel shift in the yield curve; the second factor corresponds to a "twist" in the zero curve where short maturity rates move in one direction and long maturity rates move in the opposite direction; the third factor corresponds to a "bowing" of the zero curve where short and long maturity rates move in one direction and intermediate maturity rates move in the opposite direction. After application of equation (19), the first, second, and third factors accounted for about $87 \%, 10 \%$, and $3 \%$ of the variance, respectively.

\section{Implementation of the Model}

From equation (15), the process for $F_{i}(t)$ under the measure $\mathcal{M}\left\{P\left(t, t_{m(t)}\right)\right\}$ is

$$
\frac{d F_{i}(t)}{F_{i}(t)}=\sum_{j=m(t)}^{i} \frac{\delta_{j} F_{j}(t) \sum_{q=1}^{p} \lambda_{j-m(t), q} \lambda_{i-m(t), q}}{1+\delta_{j} F_{j}(t)} d t+\sum_{q=1}^{p} \lambda_{i-m(t), q} d z_{q}
$$

or

$$
d \ln F_{i}(t)=\left[\sum_{j=m(t)}^{i} \frac{\delta_{j} F_{j}(t) \sum_{q=1}^{p} \lambda_{j-m(t), q} \lambda_{i-m(t), q}}{1+\delta_{j} F_{j}(t)}-\sum_{q=1}^{p} \frac{\lambda_{i-m(t), q}^{2}}{2}\right] d t+\sum_{q=1}^{p} \lambda_{i-m(t), q} d z_{q}
$$


An approximation that simplifies the Monte Carlo implementation of the model is that the drift of $\ln F_{i}$ is constant between times $t_{k}$ and $t_{k+1}$ so that

$$
\begin{gathered}
F_{i}\left(t_{k+1}\right)=F_{i}\left(t_{k}\right) \exp \left[\left(\sum_{j=k+1}^{i} \frac{\delta_{j} F_{j}\left(t_{k}\right) \sum_{q=1}^{p} \lambda_{j-k-1, q} \lambda_{i-k-1, q}}{1+\delta_{j} F_{j}\left(t_{k}\right)}-\sum_{q=1}^{p} \frac{\lambda_{i-k-1, q}^{2}}{2}\right) \delta_{k}\right. \\
\left.+\sum_{q=1}^{p} \lambda_{i-k-1, q} \epsilon_{q} \sqrt{\delta_{k}}\right]
\end{gathered}
$$

where $\epsilon_{q}$ are independent random samples from standard normal distributions. We can test this approximation by choosing a set of test $\Lambda_{j}$ and a test zero curve, using Monte Carlo simulation in conjunction with equation (22) to value caplets, and comparing the prices of the caplets with the exact prices given by equation (4). The $\sigma_{i}$ for equation (4) are calculated from equation (18). Each Monte Carlo trial consists of using equation (22) to generate a path for each forward rate under the $\mathcal{M}\left\{P\left(t, t_{m(t)}\right)\right\}$ measure. The value of $F_{i}\left(t_{i}\right)$ is the realized rate for the time period between $t_{i}$ and $t_{i+1}$ and enables the caplet payoff at time $t_{i+1}$ to be calculated. This payoff is discounted to time zero using $F_{j}\left(t_{j}\right)$ as the discount rate for the interval $\left(t_{j}, t_{j+1}\right)$. The estimated caplet value is the mean of the discounted payoffs.

We have carried out the test just described for a variety of term structures, volatility structures, strike rates, and number of factors. Typical results are shown in Table 1. This table is based on an upward sloping term structure, a "humped" volatility structure similar to that observed in the market, and at-the-money caplets (that is, caplets where the strike rate equals the forward rate). Three factors and 200,000 Monte Carlo trials were used. The $\delta_{i}$ were set equal to one year. ${ }^{2}$ This table shows the mean and standard error of the difference between the true spot volatility of a caplet and the volatility implied from the caplet price calculated using the LIBOR market model, with both volatilities being measured in percent. The results shown are for the fifth caplet. Similar results are obtained for other caplets, other term structures, other volatility structures, and other choices for the strike rate and the number of factors. The average interest rates reported 
in Table 1 are the arithmetic average the zero rates for maturities of $1,2,3, \ldots, 10$ years. The average volatilities are similarly the arithmetic average of the spot volatilities between one and ten years.

Table 1 shows that, for the volatility and interest rate environments that are typically encountered in North America and Europe, the implementation of the model in equation (22) gives very accurate results. Consider, for example, the case where the average interest rate is $5 \%$ and the average volatility is $20 \%$. If the true spot volatility for a particular caplet is $V \%$, Table 1 shows that we can be $95 \%$ certain that the equation (22) implementation of the LIBOR market model is implicitly assuming a spot volatility between $V-0.12 \%$ and $V+0.08 \%$. As the volatility increases the approximation in equation (22) works less well. In some countries (for example, Japan), very low short-term interest rates are sometimes coupled with spot volatilities as high as $100 \%$ for short-maturity caplets. Table 1 shows that the approximation in equation (22) may not be appropriate in this case. ${ }^{3}$ 


\section{APPROXIMATE PRICING OF EUROPEAN SWAP OPTIONS}

In this section we present an approximate, but very accurate, procedure for pricing European swap options in the LIBOR market model. Other approximations have been suggested by Brace, Gatarek, and Musiela (1997) and Andersen and Andreasen (1997). Our approach is similar in spirit to that of Andersen and Andreasen, but is much easier to implement than either of the other two approaches. It is motivated by the observation that when forward LIBOR rates are lognormal, swap rates are approximately lognormal and approximately linearly dependent on forward LIBOR rates.

As in Section I we consider an option on a swap lasting from $t_{n}$ to $t_{N+1}$ with reset dates at times $t_{n}, t_{n+1}, \ldots, t_{N}$. Initially we assume that the reset dates for the swap coincide with the reset dates for caplets underlying the LIBOR market model. Later we relax this assumption.

The relationship between bond prices and forward rates is

$$
\frac{P\left(t, t_{k}\right)}{P\left(t, t_{n}\right)}=\prod_{j=n}^{k-1} \frac{1}{1+\delta_{j} F_{j}(t)}
$$

for $k \geq n+1$. It follows that the formula for the forward swap rate in equation (6) can be written

$$
S_{n, N}(t)=\frac{1-\prod_{j=n}^{N} \frac{1}{1+\delta_{j} F_{j}(t)}}{\sum_{i=n}^{N} \delta_{i} \prod_{j=n}^{i} \frac{1}{1+\delta_{j} F_{j}(t)}}
$$

(We employ the convention that empty sums equal zero and empty products equal one.) Equivalently

$$
S_{n, N}(t)=\frac{\prod_{j=n}^{N}\left[1+\delta_{j} F_{j}(t)\right]-1}{\sum_{i=n}^{N} \delta_{i} \prod_{j=i+1}^{N}\left[1+\delta_{j} F_{j}(t)\right]}
$$

or

$$
\ln S_{n, N}(t)=\ln \left\{\prod_{j=n}^{N}\left[1+\delta_{j} F_{j}(t)\right]-1\right\}-\ln \left\{\sum_{i=n}^{N} \delta_{i} \prod_{j=i+1}^{N}\left[1+\delta_{j} F_{j}(t)\right]\right\}
$$

so that

$$
\frac{1}{S_{n, N}(t)} \frac{\partial S_{n, N}(t)}{\partial F_{k}(t)}=\frac{\delta_{k} \gamma_{k}(t)}{1+\delta_{k} F_{k}(t)}
$$


where

$$
\gamma_{k}(t)=\frac{\prod_{j=n}^{N}\left[1+\delta_{j} F_{j}(t)\right]}{\prod_{j=n}^{N}\left[1+\delta_{j} F_{j}(t)\right]-1}-\frac{\sum_{i=n}^{k-1} \delta_{i} \prod_{j=i+1}^{N}\left[1+\delta_{j} F_{j}(t)\right]}{\sum_{i=n}^{N} \delta_{i} \prod_{j=i+1}^{N}\left[1+\delta_{j} F_{j}(t)\right]}
$$

From Ito's lemma the $q$ th component of the volatility of $S_{n, N}(t)$ is

$$
\sum_{k=n}^{N} \frac{1}{S_{n, N}(t)} \frac{\partial S_{n, N}(t)}{\partial F_{k}(t)} \zeta_{k, q}(t) F_{k}(t)
$$

or

$$
\sum_{k=n}^{N} \frac{\delta_{k} \zeta_{k, q}(t) F_{k}(t) \gamma_{k}(t)}{1+\delta_{k} F_{k}(t)}
$$

The variance rate of $S_{n, N}(t)$ is therefore

$$
\sum_{q=1}^{p}\left[\sum_{k=n}^{N} \frac{\delta_{k} \zeta_{k, q}(t) F_{k}(t) \gamma_{k}(t)}{1+\delta_{k} F_{k}(t)}\right]^{2}
$$

Assuming as in Section II that $\zeta_{k, q}(t)=\lambda_{k-m(t), q}$, the variance rate of $S_{n, N}(t)$ is

$$
\sum_{q=1}^{p}\left[\sum_{k=n}^{N} \frac{\delta_{k} \lambda_{k-m(t), q} F_{k}(t) \gamma_{k}(t)}{1+\delta_{k} F_{k}(t)}\right]^{2}
$$

This expression is in general stochastic showing that when the forward rates underlying caplets are lognormal, swap rates are not lognormal. For the purposes of evaluating the expression we assume that $F_{k}(t)=F_{k}(0)$. This means that the volatility of $S_{n, N}(t)$ is constant within each accrual period and the swap rate is lognormal. The average variance rate of $S_{n, N}(t)$ between time zero and time $t_{n}$ is then

$$
\frac{1}{t_{n}} \sum_{j=0}^{n-1}\left\{\delta_{j} \sum_{q=1}^{p}\left[\sum_{k=n}^{N} \frac{\delta_{k} \lambda_{k-j-1, q} F_{k}(0) \gamma_{k}(0)}{1+\delta_{k} F_{k}(0)}\right]^{2}\right\}
$$

The spot swap option volatility is therefore

$$
\sqrt{\frac{1}{t_{n}} \sum_{j=0}^{n-1}\left\{\delta_{j} \sum_{q=1}^{p}\left[\sum_{k=n}^{N} \frac{\delta_{k} \lambda_{k-j-1, q} F_{k}(0) \gamma_{k}(0)}{1+\delta_{k} F_{k}(0)}\right]^{2}\right\}}
$$

The swap option price can be calculated by substituting this volatility into equation (10) or $(11)$. 


\section{Non-Matching Accrual Periods}

The accrual periods for the swaps underlying broker quotes for European swap options do not always match the accrual periods for the caps and floors underlying broker quotes. For example, in the United States the benchmark caps and floors have quarterly resets while the swaps underlying the benchmark European swap options have semiannual resets. To accommodate this, we now extend the analysis to the situation where each swap accrual period includes $M$ cap accrual periods where $M \geq 1$ is an integer.

Assume that $\delta_{n}, \delta_{n+1}, \ldots, \delta_{N}$ are the swap accrual periods and that within the $i$ th swap accrual period the cap accrual periods are $\epsilon_{i, 1}, \epsilon_{i, 2}, \ldots, \epsilon_{i, M}$ with

$$
\delta_{i}=\sum_{m=1}^{M} \epsilon_{i, m}
$$

Assume that $G_{i, m}(t)$ is the forward rate observed at time $t$ for the $\epsilon_{i, m}$ accrual period. Since

$$
\begin{aligned}
1+\delta_{i} F_{i}(t) & =\prod_{m=1}^{M}\left[1+\epsilon_{i, m} G_{i, m}(t)\right] \\
\frac{\partial F_{i}(t)}{\partial G_{i, m}(t)} & =\frac{\epsilon_{i, m}\left[1+\delta_{i} F_{i}(t)\right]}{\delta_{i}\left[1+\epsilon_{i, m} G_{i, m}(t)\right]}
\end{aligned}
$$

The $q$ th component of the volatility of $S_{n, N}(t)$ is

$$
\sum_{k=n}^{N} \sum_{m=1}^{M} \frac{1}{S_{n, N}(t)} \frac{\partial S_{n, N}(t)}{\partial G_{k, m}(t)} \zeta_{k, m, q}(t) G_{k, m}(t)
$$

where $\zeta_{k, m, q}$ is the $q$ th component of the volatility of $G_{k, m}(t)$. Using

$$
\frac{\partial S_{n, N}(t)}{\partial G_{i, m}(t)}=\frac{\partial S_{n, N}(t)}{\partial F_{i}(t)} \frac{\partial F_{i}(t)}{\partial G_{i, m}(t)}
$$

we find that the estimate in equation (24) of the spot swap option volatility is

$$
\sqrt{\frac{1}{t_{n}} \sum_{j=0}^{n-1}\left\{\delta_{p} \sum_{q=1}^{p}\left[\sum_{k=n}^{N} \sum_{m=1}^{M} \frac{\delta_{k} \lambda_{k-j-1, m, q} G_{k, m}(0) \gamma_{k, m}(0)}{1+\delta_{k} F_{k}(0)}\right]^{2}\right\}}
$$


where

$$
\gamma_{k, m}(t)=\gamma_{k}(t) \frac{\epsilon_{k, m}\left[1+\delta_{k} F_{k}(t)\right]}{\delta_{k}\left[1+\epsilon_{k, m} G_{k, m}(t)\right]}
$$

and $\lambda_{j, m, q}$ is the $q$ th component of the volatility of $G_{i, m}(t)$ when $i-m(t)=j$.

\section{Accuracy of Approximation}

To assess the accuracy of the assumption that forward rates are constant in equations (24) and (25) we performed tests analogous to those described in section II for testing the accuracy of caplet pricing. Typical results are shown in Table 2. These are based on 200,000 Monte Carlo trials of equation (22) using $\delta_{i}=1$, an upward sloping term structure, a "humped" volatility structure similar to that observed in the market, and three factors. The table shows the mean and standard error of the difference between the estimates of the spot volatility of swap options calculated from the LIBOR market model and estimates of the spot volatilities of swap options calculated from the approximation in Section III. The results shown are for a $5 \times 5$ swap option (that is, a five year option to enter into a five year swap) with the underlying swap being reset annually. The strike rates are at-the money (that is, the strike rate equals the forward swap rate). Similar results are obtained for other swap options, other strike rates, other interest rate term structures, other volatility structures, and other choices for the number of factors. Average interest rates and average volatilities are defined as in Table 1.

The results in Table 2 are a little more difficult to interpret than those in Table 1 . This is because we do not know what the true swap option volatility is. To obtain the "true" swap option volatility for the LIBOR market model we used Monte Carlo simulation and equation (22) to calculate the swap option price, and equation (10) to convert the price into a volatility. There are therefore three sources of error in the difference reported in Table 2: the Monte Carlo error, the error arising from the approximation in equation (22), and the error arising from the approximations in equations (24) and (25). Tests using equation (21) and large number of time steps indicate that for a given term structure and volatility structure, the errors in estimating swap option volatilities are similar in magnitude to those 
reported for caps in Table 1 . For situations where the errors in both Tables 1 and 2 are low, we can therefore assume that the approximation in Section III works well.

The results show that the approximation in Section III works well for the interest rates and volatilities normally encountered in North America and Europe. Consider, for example, the situation where interest rates average $5 \%$ and volatilities average $20 \%$. Even after the "equation (22) error" is taken into account, the error in the approximation of the $5 \times 5$ swap option volatility is likely to be less than $0.1 \%$. This translates into a pricing error of less than $\$ 2$ per $\$ 1$ million of principal. ${ }^{4}$ 


\section{MODELING VOLATILITY SKEWS}

The volatility skew for equities has been well documented by authors such as Jackwerth and Rubinstein (1996). As pointed out by Andersen and Andreasen (1997), caps and floors exhibit a similar volatility skew to equities. ${ }^{5}$ The lower the strike price, the higher the volatility implied from the standard market model in equations (4) and (5). Some brokers do provide quotes periodically for caps that are not at the money, but there is very little data on the pricing of non-at-the-money European swap options. It is, therefore, of interest to investigate how cap volatility skews can be converted to swap option volatility skews.

As shown by Andersen and Andreasen (1997), the LIBOR market model can be extended to incorporate volatility skews. We consider the version of their model where the process for forward rates in equation (12) is replaced by a CEV model

$$
d F_{i}(t)=\ldots+\sum_{q=1}^{p} \zeta_{i, q}(t) F_{i}(t)^{\alpha} d z_{q}
$$

where $\alpha$ is a positive constant. We generalize the notation in Section II to define $\lambda_{j, q}$ as the value of $\zeta_{i, q}(t)$ in this model when there are $j$ whole accrual periods between time $t$ and time $t_{i}$. This means that

$$
\zeta_{i, q}(t)=\lambda_{i-m(t), q}
$$

The process for forward rates in equation (20) becomes

$$
\frac{d F_{i}(t)}{F_{i}(t)^{\alpha}}=\sum_{j=m(t)}^{i} \frac{\delta_{j} F_{j}(t)^{\alpha} \sum_{q=1}^{p} \lambda_{j-m(t), q} \lambda_{i-m(t), q}}{1+\delta_{j} F_{j}(t)} d t+\sum_{q=1}^{p} \lambda_{i-m(t), q} d z_{q}
$$

or

$$
\begin{gathered}
d Q_{i}(t)=\sum_{j=m(t)}^{i}\left[\frac{\delta_{j} F_{j}(t)^{\alpha} \sum_{q=1}^{p} \lambda_{j-m(t), q} \lambda_{i-m(t), q}}{1+\delta_{j} F_{j}(t)}-\sum_{q=1}^{p} \frac{\alpha F_{i}(t)^{\alpha-1} \lambda_{i-m(t), q}^{2}}{2}\right] d t \\
+\sum_{q=1}^{p} \lambda_{i-m(t), q} d z_{q}
\end{gathered}
$$

where

$$
Q_{i}(t)=\frac{1}{1-\alpha} F_{i}(t)^{1-\alpha}
$$


Analogously to the approach taken in section II in arriving at equation (22), we assume that the drift of $Q_{i}(t)$ is constant between times $t_{j}$ and $t_{j+1}$ to get

$$
\begin{gathered}
Q_{i}\left(t_{k+1}\right)=Q_{i}\left(t_{k}\right)+\delta_{k} \sum_{j=k+1}^{i}\left[\frac{\delta_{j} F_{j}\left(t_{k}\right)^{\alpha} \sum_{q=1}^{p} \lambda_{j-k-1, q} \lambda_{i-k-1, q}}{1+\delta_{j} F_{j}\left(t_{k}\right)}-\sum_{q=1}^{p} \frac{\alpha F_{i}\left(t_{k}\right)^{\alpha-1} \lambda_{i-k-1, q}^{2}}{2}\right] \\
+\sum_{q=1}^{p} \lambda_{i-k-1, q} \epsilon_{q} \sqrt{\delta_{k}}
\end{gathered}
$$

We define $\Lambda_{j}$ by

$$
\Lambda_{j}=\sqrt{\sum_{q=1}^{p} \lambda_{j, q}^{2}}
$$

and $\sigma_{i}$ by:

$$
\sigma_{i}^{2}=\frac{1}{t_{i}} \sum_{j=1}^{i} \Lambda_{i-j}^{2} \delta_{j-1}
$$

These definitions are consistent with equations (17) and (18) in Section II. However, our notation is now more general than in Section II. In equations (17) and (18) the $\Lambda$ 's and $\sigma$ 's were volatilities whereas here they are merely volatility parameters.

Andersen and Andreasen (1997) show that the process in equation (26) implies that the price of the $i$ th caplet is

$$
P\left(0, t_{i+1}\right) L_{c} \delta_{i}\left[F_{i}(0)-F_{i}(0) \chi^{2}(a, b+2, c)-R_{c} \chi^{2}(c, b, a)\right]
$$

when $0<\alpha<1$ and

$$
P\left(0, t_{i+1}\right) L_{c} \delta_{i}\left[F_{i}(0)-F_{i}(0) \chi^{2}(c,-b, a)-R_{c} \chi^{2}(a, 2-b, c)\right]
$$

when $\alpha>1$ where

$$
a=\frac{R_{c}^{2(1-\alpha)}}{(1-\alpha)^{2} \sigma_{i}^{2} t_{i}} ; \quad b=\frac{1}{1-\alpha} ; \quad c=\frac{F_{i}(0)^{2(1-\alpha)}}{(1-\alpha)^{2} \sigma_{i}^{2} t_{i}}
$$

and $\chi^{2}(z, v, k)$ is the cumulative probability that a non-central chi-squared distribution with non-centrality parameter $v$ and $k$ degrees of freedom is less than $z$. The price of the $i$ th floorlet is

$$
P\left(0, t_{i+1}\right) L_{c} \delta_{i}\left[R_{c}-F_{i}(0) \chi^{2}(a, b+2, c)-R_{c} \chi^{2}(c, b, a)\right]
$$


when $0<\alpha<1$ and

$$
P\left(0, t_{i+1}\right) L_{c} \delta_{i}\left[R_{c}-F_{i}(0) \chi^{2}(c,-b, a)-R_{c} \chi^{2}(a, 2-b, c)\right]
$$

when $\alpha>1$. When $\alpha=1$, equations (4) and (5) give the caplet and floorlet prices, respectively.

Andersen and Andreasen (1997) also show that skews for swap rates can be analyzed in an analogous way to skews for forward rates. Suppose that the swap rate considered in Section I follows a process of the form

$$
d S_{n, N}(t)=\ldots+\sum_{q=1}^{p} \eta_{q, n, N}(t) S_{n, N}(t)^{\beta} d z_{q}
$$

where $\beta$ is a positive constant. Generalizing our earlier definition of $\sigma_{n, N}$, we set:

$$
\sigma_{n, N}^{2}=\frac{1}{t_{n}} \int_{\tau=0}^{t_{n}} \sum_{q=0}^{p} \eta_{q, n, N}(\tau)^{2} d \tau
$$

(In the special case where $\beta=1$ this is the same as the $\sigma_{n, N}$ in Section I.) The value of the European swap option that gives the holder the right to pay fixed is

$$
L_{s} A_{n, N}(0)\left[S_{n, N}(0)-S_{n, N}(0) \chi^{2}(e, f+2, g)-R_{s} \chi^{2}(g, f, e)\right]
$$

when $0<\alpha<1$ and

$$
L_{s} A_{n, N}(0)\left[S_{n, N}(0)-S_{n, N}(0) \chi^{2}(g,-f, e)-R_{s} \chi^{2}(e, 2-f, g)\right]
$$

when $\alpha>1$ where

$$
e=\frac{R_{s}^{2(1-\beta)}}{(1-\beta)^{2} \sigma_{n, N}^{2} t_{n}} ; \quad f=\frac{1}{1-\beta} ; \quad g=\frac{S_{n, N}(0)^{2(1-\beta)}}{(1-\beta)^{2} \sigma_{n, N}^{2} t_{n}}
$$

The value of the European swap option that gives the holder the right to pay floating is

$$
L_{s} A_{n, N}(0)\left[R_{s}-S_{n, N}(0) \chi^{2}(e, f+2, g)-R_{s} \chi^{2}(g, f, e)\right]
$$


when $0<\alpha<1$ and

$$
L_{s} A_{n, N}(0)\left[R_{s}-S_{n, N}(0) \chi^{2}(g,-f, e)-R_{s} \chi^{2}(e, 2-f, g)\right]
$$

when $\alpha>1$. When $\beta=1$, equations (10) and (11) give the swap option price.

In Section III we showed that, for the volatility and interest rate environments normally encountered, when $\alpha=1$ in equation (26) it is reasonable to assume that swap rates follow the process in equation (32) with $\beta=1$. We now hypothesize that a more general version of this result holds. We suppose that, when rates follow the process in equation (26), to a reasonable approximation swap rates follow the process in equation (32) with $\beta=\alpha$. This is an attractive hypothesis. A similar analysis to that in Section III shows that it leads to $\sigma_{n, N}$ being approximately equal to ${ }^{6}$

$$
S_{n, N}(0)^{1-\beta} \sqrt{\frac{1}{t_{n}} \sum_{j=0}^{n-1}\left\{\delta_{p} \sum_{q=1}^{p}\left[\sum_{k=n}^{N} \sum_{m=1}^{M} \frac{\delta_{k} \lambda_{k-j-1, m, q} G_{k, m}(0)^{\alpha} \gamma_{k, m}(0)}{1+\delta_{k} F_{k}(0)}\right]^{2}\right.}
$$

where $S_{n, N}(0)$ is given by equation $(23)$.

To test the hypothesis we compared European swap option prices calculated from:

1. A Monte Carlo simulation of the extended LIBOR market model using the approximation in equation (27); and

2. Equation (35) or (36) combined with the estimate of $\sigma_{n, N}$ in equation (37) and $\beta=\alpha$. Typical results, based on 100,000 antithetic simulation trials, are shown in Table 3. These are for a $3 \times 3$ European swap option in which the holder has the right to pay floating. The average ten year interest rate is $5 \%$ and the average ten year volatility is 20\%. The term structure is upward sloping and the volatility structure is humped. The tenor of the caplets underlying the model is three months and the swap underlying the European swap option is reset semiannually.

The results for $\alpha \neq 1$ are not quite as good as those for $\alpha=1$, but they are still quite acceptable. The largest errors (about $0.4 \%$ ) are for deep-in-the-money swap options. The 
prices of these options are relatively insensitive to volatilities and so these errors are not a cause for serious concern.

Figures 1 and 2 use the results in this section to compare cap volatility skews with swap option volatility skews in a variety of situations. The figures show flat volatilities for a five year cap and spot volatilities for $3 \times 3$ European swap option. In Figure 1 the term structure is flat at 5\%; in Figure 2 it is upward sloping. Three different values of $\alpha$ are considered. The cap is reset quarterly; the swap underlying the swap option is reset semiannually. In Figures 1(a) and 2(a) the volatility structure is flat at 20\%. In Figures 1(b) and 2(b) it has a hump similar to that observed in the market.

The figures show that care must be exercised in interpreting cap volatility skews. The latter are affected by the way in which the flat volatilities quoted by brokers are calculated. A flat volatility is the volatility that, when applied to all caplets, produces the market price. It is, therefore, a complex non-linear function of the caplet spot volatilities. As shown by the cap volatilities for the $\alpha=1$ case in Figure $1(\mathrm{~b})$, the function depends on the strike price. (If the function did not depend on the strike price, these cap volatilities would be the same for all strike prices.)

When the term structure is upward sloping (Figure 2), the cap results are affected by the fact that the moneyness of the early caplets is less than that of the later caplets. Consider, for example, the case where the volatility structure is flat and the cap is at the money (Figure 2a). The short maturity caplets are out of the money and the long maturity caplets are in the money. When $\alpha=1$ they are all priced with the same volatility. When $\alpha=0.5$, the short maturity caplets are priced with relatively low volatilities and long maturity caplets are priced with relatively high volatilities. This increases the value of the cap. When $\alpha=1.5$ the short maturity caplets are priced with relatively high volatilities and long maturity caplets are priced with relatively low volatilities. This decreases the value of the cap.

The figures show that, once an appropriate model has been chosen $3 \times 3$ swap option 
volatilities are relatively insensitive to the shape of the volatility term structure. Implied volatilities are slightly higher for the humped volatility structure. This is simply a reflection of the fact the volatilities during the first three years are slightly higher for the humped volatility structure we used than for a flat $20 \%$ volatility structure. 


\section{APPLICATIONS OF THE MODEL}

There are two main applications of the model in Section IV. The first is to valuation and marking to market of non-standard interest rate derivatives. The second is to the valuation and marking to market of non-at-the-money European swap options. We will discuss both of these applications in this section. We will use for illustration U.S. data on caps and European swap options for August 12, 1999, kindly supplied to us by a major investment bank. The data were compiled by averaging mid-market quotes from a number of different brokers. ${ }^{7}$ It was used by the bank to mark to market its portfolio of caps and European swap options on August 12, 1999. The cap data consist of 133 flat volatility quotes where the strike prices range from $3 \%$ to $10 \%$ and the cap lives range from one to ten years. The swap data consist of at-the-money volatility quotes for a range of different option maturities and swap lives. The caps were reset quarterly and the swaps were reset semiannually. The zero curve on August 12, 1999 was upward sloping with short rates approximately $5.3 \%$ and long rates approximately $7.2 \%$.

The first step in implementing the model in Section IV is to estimate $\alpha$. This can be done by simultaneously estimating $\alpha$ and the $\Lambda_{j}$ from cap prices. ${ }^{8}$ We search for the values of $\alpha$ and the $\Lambda_{j}$ that minimize the root mean square cap pricing error

$$
\sqrt{\frac{1}{K} \sum_{i=1}^{K}\left(u_{i}-v_{i}\right)^{2}}
$$

where $K$ is the number of caps for which market data is available, $u_{i}$ is the market price of the $i$ th cap calculated from the broker (flat) volatility quotes using equation (4), and $v_{i}$ is the model price of the $i$ th cap calculated using equations (30) and (31).

The best fit value of $\alpha$ on August 12, 1999 was found to be 0.716. This value of $\alpha$ reflects a situation where, for caps with maturities of three years or more, quoted volatilities declined in absolute terms by between $2 \%$ to $4 \%$ as the strike price increased from $3 \%$ to $10 \%$. The best fit values of the $\Lambda_{j}$ obtained from this analysis of cap prices are shown in the Caps column of Table 4. As in the case of the lognormal model discussed in Section 
II, we assumed seven different levels for $\Lambda$ (see footnote 2) and imposed the smoothness constraint that $\Lambda_{j}$ be a non-increasing function of $j$ for $j \geq 11$.

A natural application of the LIBOR market model, once it has been calibrated to plain vanilla caps in the way just described, is to the valuation of non-standard cap products. Hull (2000) examines three such products: ratchet caps, sticky caps, and flexi caps. In a ratchet cap the strike rate for a caplet equals the LIBOR rate at the previous reset date plus a spread. In a sticky cap it equals the previous capped LIBOR rate plus a spread. In a flexi cap there is an upper bound to the total number of caplets that are exercised. Hull finds that the prices of all three types of nonstandard caps are dependent on the number of factors. This is because their payoffs, unlike those of a plain vanilla cap, depend on the joint behavior of two or more forward rates. Hull's analysis used Monte Carlo simulation in conjunction with equation (22) to price the instruments.

To value non-standard swap option products, such as Bermudan swap options, the most appropriate procedure would seem to be to set $\alpha$ equal to its best fit value calculated from caps and choose the $\Lambda_{j}$ so that they fit broker quotes on at-the-money European swap options. We did this using 30 swap option quotes where the option maturity ranged from 0.5 years to 5 years and the swap life ranged from 1 to 5 years. We used a three factor model, determining the $\lambda_{j, q}$ from the $\Lambda_{j}$ as indicated in equation (19). The results are shown in the Swap Option column of Table 4.

If the three-factor extended LIBOR market model were perfect, we would of course obtain the same parameter values regardless of the calibrating instruments being used. The reality is that all derivative models - even those involving several factors - have to be fine tuned to reflect the use to which they are being put. Table 4 shows that the $\Lambda_{j}$ values obtained by fitting the model to swap options are different from those obtained by fitting it to caps. Specifically, the $\Lambda$ 's implied from swap options are less humped than those implied from caps. This appears to be because the pricing of options on one and two year swaps does not fully reflect the hump observed in cap volatilities. 
The root mean square pricing error for caps in Table 4 is greater than that for swap options. This reflects the fact that we are fitting the model to 133 caps and only 30 swap options. The relatively high average absolute percentage error for caps reflects both this and the existence of some errors in deep-out-of-the-money caps that, although small in absolute terms, were large when measured as percentages.

\section{Incorporating Volatility Skews when European Swap Options are Priced}

As already mentioned broker quotes are available only for at-the-money European swap options. This creates problems for financial institutions when they price and mark to market non-at-the-money swap options. Given the volatility skew in the cap market it is unlikely to be correct to assume the same volatility for all strike prices in the European swap option market.

The analysis in Section IV makes it possible to calculate a complete volatility skew for European swap options from broker quotes on at-the-money instruments. The procedure is as follows.

1. Calculate the price of the at-the-money swap option from its volatility using equation (10) or (11)

2. Imply a value of $\sigma_{n, N}$ from the price of the at-the-money European swap option using equation (33) or (35) with $\alpha$ set equal to its best fit value

3. Use this value of $\sigma_{n, N}$ to calculate the price of non-at-the-money European swap option using equation (33) or (35)

4. Imply a Black volatility for the non-at-the-money swap options using equation (10) or $(11)^{9}$

As an example we consider a $5 \times 5$ European swap option on August 12, 1999. (For the purposes of calculating the volatility skew it does not matter whether it is an option to receive fixed or receive floating.) The broker quote for the volatility of this instrument is $17.58 \%$ and the at-the-money strike swap rate is $7.47 \%$. The results of the above procedure are displayed in Figure 3. 
Note that the calculation of a volatility skew for European swap options depends only on the best fit value of $\alpha$ ( 0.716 for the case considered) and the at-the-money volatilities. It does not require an estimate of the $\Lambda_{j}$ or the $\lambda_{j, q}$. It should be relatively easy for financial institutions to store a value of $\alpha$ and update it periodically so that very fast volatility skew calculations can be made whenever required. 


\section{CONCLUSIONS}

Caps and European swap options are quite different instruments. A cap is a portfolio of options; a European swap option is an option on an annuity. This paper has provided a simple robust procedure for relating the volatilities used by the market to price European swap options to the volatilities used by the market to price caps. Given the popularity of caps and European swap options, the procedure presents traders with opportunities to fine tune their pricing and search for arbitrage opportunities. A key contribution of the results in the paper is to make it very easy for traders to quickly calculate volatility skews for European swap options (which are not provided by brokers) from volatility skews for caps (which are provided by brokers).

The model underlying our results is the multifactor extended LIBOR market model proposed by Andersen and Andreasen (1997). The results we have produced make it possible to calibrate the model very quickly to either caps or European swap options. Non-standard European-style options can be easily priced using the model. New numerical procedures developed by authors such as Andersen (1998), Broadie and Glasserman (1997), and Longstaff and Schwartz allow American-style non-standard instruments to be handled. 


\section{REFERENCES}

Andersen, L. "A Simple Approach to the Pricing of Bermudan Swaptions in the Multifactor LIBOR Market Model," Working paper, Gen Re Financial Products, 1998, forthcoming, Journal of Computational Finance.

Andersen L. and J. Andreasen, "Volatility Skews and Extensions of the LIBOR Market Model," Working paper, Gen Re Financial Products, 1997, forthcoming, Applied Mathematical Finance.

Black, F. "The Pricing of Commodity Contracts," Journal of Financial Economics, 3 (March 1976), 167-79.

Brace, A, D. Gararek, and M. Musiela. "The Market Model of Interest Rate Dynamics," Mathematical Finance, 7, 2 (1997), 127-55.

Broadie, M. and P. Glasserman. "A Stochastic Mesh Method for Pricing High Dimensional American Options," Working Paper, Columbia University, New York, 1997.

Harrison, J. M. and D. M. Kreps. "Martingales and Arbitrage in Multiperiod Securities Markets," Journal of Economic Theory, 20 (1979), 381-408.

Heath, D., R. Jarrow, and A. Morton. "Bond Pricing and the Term Structure of Interest Rates: A New Methodology," Econometrica, 60, 1 (1992), 77-105.

Hull, J. C. Options, Futures, and Other Derivatives, Fourth Edition, Prentice Hall, Englewood Cliffs, NJ, 2000.

Jackwerth, J.C. and M. Rubinstein, "Recovering Probability Distributions from Option Prices," Journal of Finance, 51 (December 1996), 1611-31

Jamshidian, F. "The LIBOR and Swap market Models," Finance and Stochastics, 1 (1997), 293-330.

Longstaff, F. and E. Schwartz. "Valuing American Options by Simulation: A Least Squares Approach," Working Paper 25-98, 1998, Andersen School at UCLA.

Miltersen, K., K. Sandmann, and D. Sondermann. "Closed Form Solutions for Term Structure Derivatives with Lognormal Interest Rates," Journal of Finance, 52, 1 (March 1997), 409-30.

Rebonato, R. "On the Simultaneous Calibration of Multifactor Lognormal Interest rate Models to Black Volatilities and to the Correlation Matrix," Journal of Computational Finance, 2, 4 (Summer 1999), 5-27. 


\section{Table 1}

\section{Typical Results for Accuracy of Caplet Volatilities}

The table shows the amount by which the true spot caplet volatility exceeds the volatility given by the Monte Carlo implementation of the LIBOR market model in equation (22). For example, when the average rate is $1 \%$, the average volatility is $50 \%$, and the true caplet volatility is $V \%$, the Monte Carlo simulation produces a price consistent with a volatility of $V+0.08 \%$. The standard error of the volatility difference is in parentheses. Results are for 200,000 antithetic simulation trials, an upward sloping term structure, and a humped volatility structure. The caplet has a maturity of five years and tenor of one year. All volatilities are measured as percentages.

\begin{tabular}{ccccc}
\hline $\begin{array}{c}\text { Average } \\
\text { Rate }\end{array}$ & $10 \%$ & $20 \%$ & $50 \%$ & $100 \%$ \\
\hline $1 \%$ & $-0.01(0.02)$ & $-0.01(0.05)$ & $-0.08(0.25)$ & $0.25(1.19)$ \\
$5 \%$ & $-0.02(0.02)$ & $-0.02(0.05)$ & $-0.56(0.17)$ & $2.57(0.60)$ \\
$10 \%$ & $-0.02(0.02)$ & $-0.01(0.04)$ & $-0.02(0.12)$ & $9.42(0.44)$ \\
$20 \%$ & $-0.01(0.02)$ & $-0.02(0.03)$ & $0.39(0.08)$ & $14.64(0.43)$ \\
$50 \%$ & $0.00(0.01)$ & $0.01(0.02)$ & $1.65(0.11)$ & $14.55(0.52)$ \\
& & & & \\
\hline
\end{tabular}


Table 2

\section{Typical Results for Accuracy of Swap Option Volatilities}

The table shows the amount by which $5 \times 5$ swap option spot volatilities, calculated using the approximation in Section III, exceed those calculated using the Monte Carlo implementation of the LIBOR market model in equation (22). For example, when the average rate is $1 \%$, the average volatility is $50 \%$, and the swap option volatility calculated from the approximation in Section III is $V \%$, the Monte Carlo simulation produces a price consistent with a volatility of $V-0.05 \%$. The standard error of the Monte Carlo estimate is in parentheses. Results are for 200,000 antithetic simulation trials, an upward sloping term structure, and a humped volatility structure. Both the caps underlying the LIBOR market model and the swaps underlying the swap option are reset annually. All volatilities are measured as percentages.

\begin{tabular}{ccccc}
\hline $\begin{array}{c}\text { Average } \\
\text { Rate }\end{array}$ & $10 \%$ & $20 \%$ & $50 \%$ & $100 \%$ \\
\hline $1 \%$ & $-0.01(0.01)$ & $-0.02(0.02)$ & $0.05(0.02)$ & $1.00(0.06)$ \\
$5 \%$ & $-0.01(0.01)$ & $0.00(0.02)$ & $0.14(0.04)$ & $3.29(0.05)$ \\
$10 \%$ & $0.00(0.02)$ & $0.02(0.03)$ & $0.34(0.08)$ & $5.11(0.16)$ \\
$20 \%$ & $-0.01(0.02)$ & $0.04(0.04)$ & $1.01(0.14)$ & $4.13(0.35)$ \\
$50 \%$ & $0.01(0.03)$ & $0.24(0.07)$ & $-0.65(0.35)$ & $-71.24(4.13)$ \\
\hline
\end{tabular}


Table 3

\section{Typical Results for Accuracy of Swap Option Volatilities When There is a Volatility Skew}

The table shows the amount by which $3 \times 3$ swap option volatilities calculated from the approximation in Section IV exceed those calculated using the Monte Carlo implementation of the extended LIBOR market model in equation (27). For example, when $\alpha$ is 0.50 , the strike rate is $80 \%$ of the forward par yield, and the lognormal swap volatility calculated using Section IV is $V \%$, the Monte Carlo simulation produces a price consistent with a lognormal volatility of $V-0.19 \%$. The standard error of the Monte Carlo price is in parentheses. Results are for 100,000 antithetic simulation trials, an upward sloping term structure and a humped volatility structure. Caps used to define the LIBOR market model are reset quarterly and the swap underlying the swap option is reset semiannually. All volatilities are measured as percentages.

\begin{tabular}{cccccc}
\hline \multicolumn{5}{c}{ Strike Rate $\%$ of Forward Par Yield $)$} \\
$\alpha$ & 60 & 80 & 100 & 120 & 140 \\
\hline 0.10 & $0.15(0.05)$ & $0.03(0.05)$ & $-0.03(0.04)$ & $-0.02(0.04)$ & $-0.01(0.05)$ \\
0.25 & $0.34(0.05)$ & $0.14(0.04)$ & $-0.11(0.04)$ & $-0.11(0.04)$ & $-0.01(0.04)$ \\
0.50 & $0.38(0.04)$ & $0.19(0.04)$ & $0.09(0.04)$ & $0.09(0.03)$ & $0.12(0.03)$ \\
0.75 & $0.17(0.04)$ & $0.05(0.04)$ & $0.01(0.04)$ & $0.01(0.04)$ & $0.05(0.02)$ \\
1.00 & $0.09(0.04)$ & $0.02(0.04)$ & $-0.01(0.04)$ & $0.01(0.03)$ & $0.06(0.02)$ \\
& & & & & \\
1.50 & $0.21(0.04)$ & $0.04(0.04)$ & $0.01(0.03)$ & $0.05(0.02)$ & $0.09(0.02)$ \\
\hline
\end{tabular}




\section{Table 4}

Best Fit Values of Volatility Parameters on August 12, 1999

The table shows best fit values for the volatility parameters, $\Lambda_{j}$ when the model defined by equations (26) to (28) is fitted to 133 caps (column 2) and 30 European swap options (column 3). The CEV parameter $\alpha$ was set equal to its best fit value of 0.716 . Principal $=\$ 1,000$ and $\delta_{j}=0.25$ for all $j$.

\begin{tabular}{|c|c|c|}
\hline & \multicolumn{2}{|c|}{ Values of $\Lambda_{j}$ for } \\
\hline & Caps & Swap Options \\
\hline $1 \leq j \leq 3$ & 0.0795 & 0.0866 \\
\hline $4 \leq j \leq 7$ & 0.1300 & 0.1076 \\
\hline $8 \leq j \leq 11$ & 0.1274 & 0.0890 \\
\hline $12 \leq j \leq 15$ & 0.0795 & 0.0890 \\
\hline $16 \leq j \leq 19$ & 0.0756 & 0.0890 \\
\hline $20 \leq j \leq 27$ & 0.0756 & 0.0808 \\
\hline $28 \leq j \leq 39$ & 0.0756 & 0.0730 \\
\hline RMS Pricing Error $(\$)$ & 0.57 & 0.21 \\
\hline Mean Abs \% Pricing Error & 5.72 & 1.27 \\
\hline
\end{tabular}


(a)

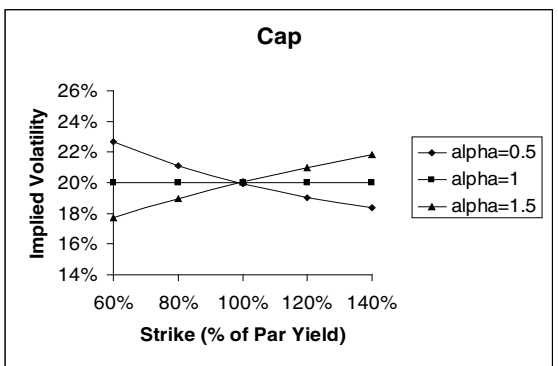

(b)

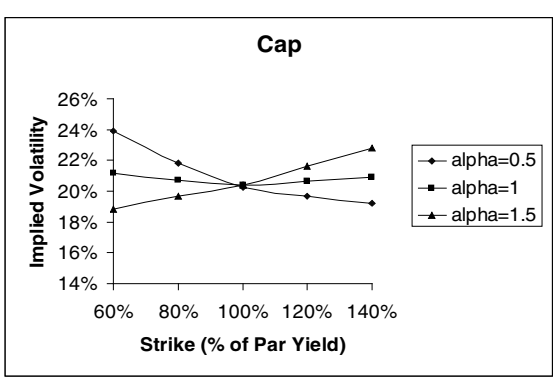

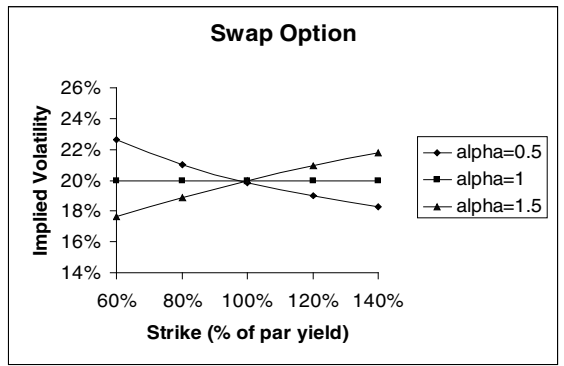

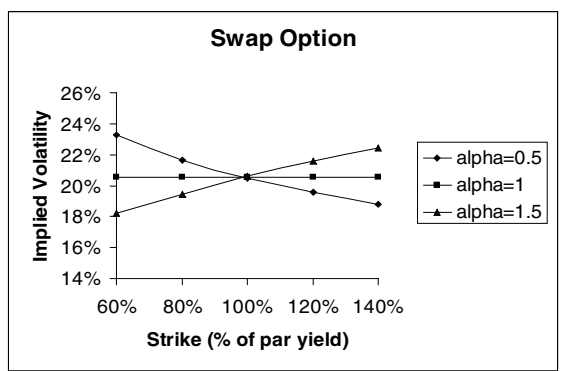

Figure 1

Volatility skews for a flat term structure. The cap is reset quarterly and has a life of 3 years. The European swaption is a 3 year option into a 3 year swap that is reset semiannually. Results are based on the model in Section IV. In (a) the volatility structure is flat. In (b) it is humped. 
(a)

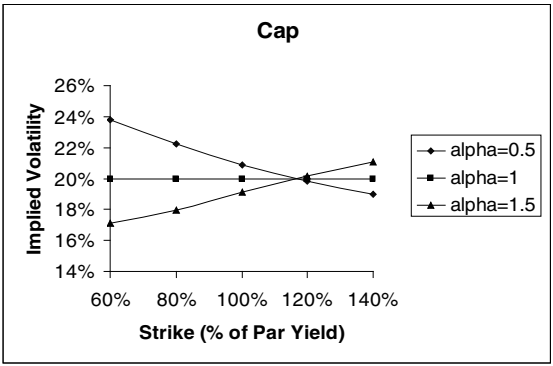

(b)

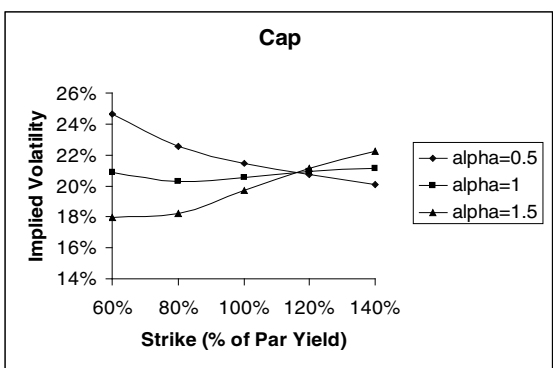

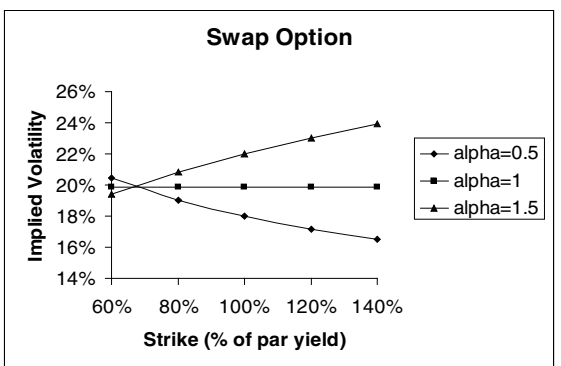

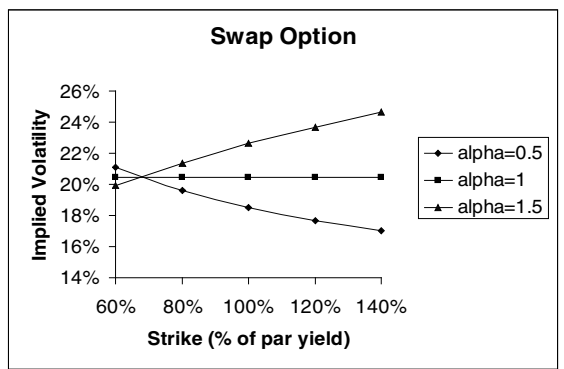

Figure 2

Volatility skews for a upward sloping term structure. The cap is reset quarterly and has a life of 3 years. The European swaption is a 3 year option into a 3 year swap that is reset semiannually. Results are based on the model in Section IV. In (a) the volatility structure is flat. In (b) it is humped. 


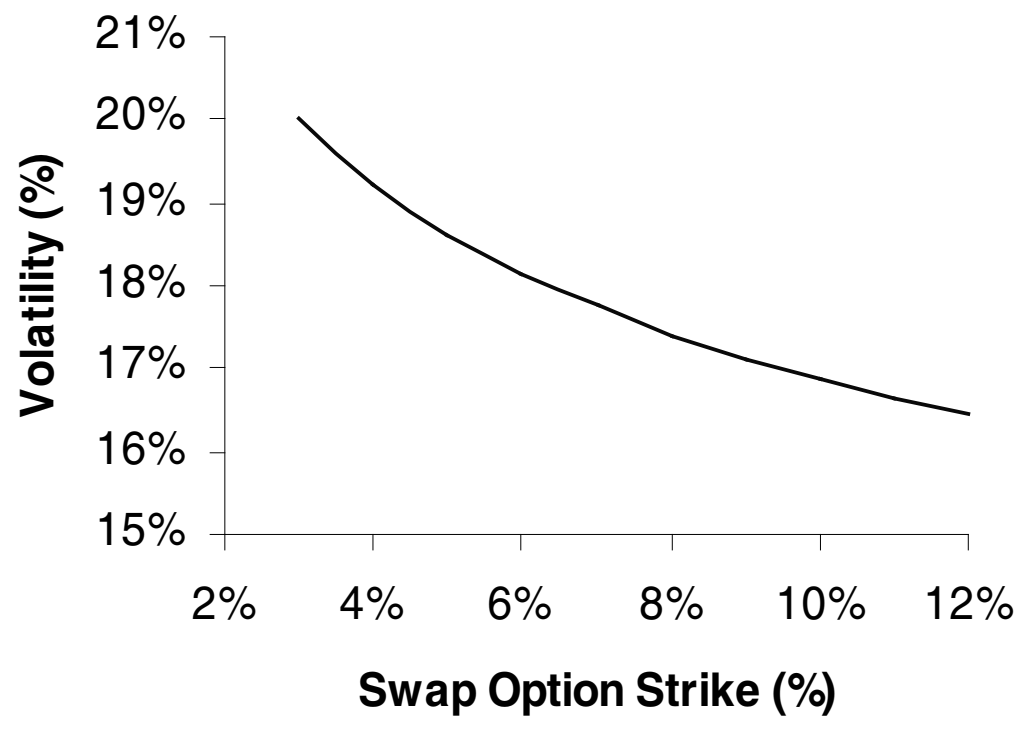

Figure 3

Volatility skew for $5 \times 5$ swap options on August 12, 1999. This is based on a value of 0.716 for the $\mathrm{CEV}$ parameter, $\alpha$ (estimated from cap data) and a broker quote of $17.58 \%$ for the volatility of an at-the-money $5 \times 5$ swap option 


\section{ENDNOTES}

1. Other approaches, involving the inclusion in the objective function of penalties for changes in the $\Lambda$ 's, can also be used

2. The error in equation (22) is likely to increase with the length of the accrual period. Setting the accrual period equal to one year therefore provides a relatively tough test of the approximation.

3. The lognormal LIBOR market model described here may itself be inappropriate in very-low interest rate environments. This is because it assumes a stationary volatility structure. When short rates are in the region of $1 \%$, their volatilities are very high. But, if as time passes short rates increase, more normal volatilities are likely to be observed.

4. Brace, Gatarek, and Musiela (1997) report errors for a $5 \times 5$ swap option of about $\$ 5$ per $\$ 1$ million of principal using their approximation. Although their errors are higher than ours, they are acceptably low. The chief advantage of our approach over theirs is that it is much easier to implement.

5. To be precise, the predominant pattern in the implied volatilities appears to be a skew. For short maturity caps there is a tendency for the implied volatility to start increasing when the strike price.

6. A more general version of the hypothesis is that swap rates follow the process in equation (32) for some value of $\beta$ not necessarily equal to $\alpha$. We tested whether we should use the more general hypothesis by defining a "best fit" value of $\beta$ as the value for which the expression in equation (37) is least sensitive to perturbations in forward rates. In a wide range of situations this value of $\beta$ was found to be very close to $\alpha$. Encouragingly, the best fit value of $\beta$ also led to the expression in equation (37) being almost exactly constant.

7. Because different brokers quote data in different ways, some judgment must be used in averaging quotes.

8. Note that cap prices depend only on the $\Lambda_{j}$ in equation (28) - not on the way in which $\Lambda_{j}$ is divided into its component parts, $\lambda_{j, q}$.

9. In theory, this last step is unnecessary since the third step provides the price. In practice it is likely to be desirable as most traders and analysts naturally think in terms of Black volatilities. 